\title{
DISTOCIA EM CABRA MESTIÇA ANGLO-NUBIANA
}

Kelry Mayara Silva ${ }^{1}$, Paulo Henrique Yamada ${ }^{1}$, Juliana de Oliveira Bernardo ${ }^{1}$, Dayrine Candido Santos ${ }^{1}$, Ariadne Pegoraro Mastelaro ${ }^{2}$, Whelerson Luiz Vitro ${ }^{3}$, Ariane Dantas ${ }^{1}$, Eunice Oba ${ }^{1}$

${ }^{1}$ Universidade Estadual Paulista - UNESP, Faculdade de Medicina Veterinária e Zootecnia - FMVZ, Botucatu, SP.

${ }^{2}$ Universidade Federal do Paraná - UFPR. ${ }^{3}$ Faculdade de Ciências Agrárias de Andradina - FCAA, Curso de Medicina Veterinária, Andradina, SP. E-mail: kelrymy@hotmail.com

\section{RESUMO}

O objetivo deste trabalho foi relatar o procedimento de cesariana em um caso de parto distócico em caprino. Uma cabra mestiça Anglo-Nubiana de quatro anos de idade, pesando 40kg foi atendida no Hospital Veterinário Antônio Francisco Fonzar da Faculdade de Ciências Agrárias de Andradina, exibindo dificuldade ao parto. Ao exame físico, o animal apresentava-se inquieto, com apetite presente e bom estado corporal. No exame vaginal realizado foi constado prolapso vaginal, retal, dilatação cervical insuficiente, porém, sem observação de feto e membranas fetais. No exame clínico verificou-se que a temperatura retal e frequência cardíaca e respiratória estavam dentro dos limites fisiológicos para a espécie. Realizou-se uma ultrassonografia uterina observando gestação gemelar, viabilidade fetal e apresentação fetal anormal devido a posição transversal de um dos fetos. O plano terapêutico escolhido foi a realização de cesariana, o tratamento pós-operatório consistiu na administração de Flunixin Meglumina ( $2 \mathrm{mg} / \mathrm{kg}$ de peso corporal, durante 3 dias), Oxitetraciclina $20 \%$ (20 mg/kg de peso vivo, em uma única aplicação) e Penicilina Benzatina (20.000 Ul/kg a cada 48 horas, totalizando 5 aplicações). Para o curativo local utilizou-se pomada a base de gentamicina, sulfadiazina de prata, vitamina $A$ e unguento. Após duas semanas o animal já apresentava plena recuperação, conclui-se que a conduta e os procedimentos cirúrgico foram bem-sucedidos.

Palavras-chave: cesariana; obstetrícia; parto distócico.

\section{DYSTOCIA IN GOAT MESTIÇA ANGLO-NUBIANA}

\begin{abstract}
The objective of this study was to report the cesarean section procedure in a case of dystocic delivery in goats. A four-year-old Anglo-Nubian goat, weighing $40 \mathrm{~kg}$, was attended at the Veterinary Hospital Antônio Francisco Fonzar of the Faculty of Agrarian Sciences of Andradina, presenting difficulty in parturition. On physical examination, the animal was restless, with a present appetite and good body condition. The vaginal examination revealed vaginal prolapse, rectal, insufficient cervical dilatation, but without fetal observation and fetal membranes. In the clinical examination it was verified that the rectal temperature and heart and respiratory rate were within the limits considered normal for the species. A uterine ultrasound was performed observing twin pregnancy, fetal viability and abnormal fetal presentation due to the transverse position of one fetus. The therapeutic plan chosen for cesarean section, the postoperative treatment consisted of administration of Flunixin meglumine $(2 \mathrm{mg} / \mathrm{kg}$ body weight for 3 days), Oxytetracycline $20 \%$ (20 mg/kg body weight in a single application) and Benzathine Penicillin $(20,000 \mathrm{IU} / \mathrm{kg}$ every 48 hours, totaling 5 applications). For the local dressing was used ointment based on gentamicin, silver sulfadiazine, vitamin A and ointment. After two weeks the animal already had full recovery, it is concluded that the surgical procedures and conduct were successful.
\end{abstract}

Keywords: caesarean; obstetrics; dystocic delivery.

\section{INTRODUÇÃO}

O desenvolvimento de um parto normal depende da interação de diversos fatores que alteram a morfologia e a fisiologia da fêmea 
gestante. Qualquer alteração nestes mecanismos pode desencadear o bloqueio ou interrupção do parto, colocando em risco vida da mãe e da cria. Estes eventos, onde há dificuldade no parto, são caracterizados como distocias, podendo ser classificadas de acordo com a sua origem (BLOOD et al., 2011).

A distocia em pequenos ruminantes é ocasionada por diferentes fatores, podendo ser fetal ou materno. A fetal ocorre devido hipertrofia, alteração de estática fetal, parto gemelar e mal formações, já a materna pode ser caracterizada por atonia ou hipertonia uterina, alterações das vias fetais moles e estreitamento de vias fetais ósseas (PUGH; BAIRD, 2012). Dessa forma, a capacidade de distinguir as dificuldades de parto é importante, pois está diretamente relacionado com o tipo de tratamento a ser executado e consequentemente com o sucesso da assistência obstétrica realizada.

Embora a incidência de distocia em pequenos ruminantes seja mundialmente baixa (<5\%) (BHATTACHARYYA et al., 2015), o conhecimento da casuística e de condutas terapêuticas é fundamental, visto que o risco de perder o filhote, bem como a mãe aumenta com o atraso do tratamento, podendo causar metrite necrótica, geralmente fatal (CHRISTOS et al., 2012).

A extração manual do feto pode ser praticada na maioria dos casos, no entanto, a intervenção cirúrgica precoce por meio da cesariana foi relatada como um método efetivo para o tratamento de distocia em ruminantes, permitindo na maioria das vezes obtenção satisfatória de recuperação da parturiente, nascimento de filhote vivo e saudável (MAJEED et al., 1993).

Contudo, não há artigos de revisão recentes na literatura que resumam os resultados de relatos de casos publicados e ensaios clínicos sobre indicações. Há sim, abordagens cirúrgicas, procedimentos e resultados após cesariana em ovinos e caprinos (ISMAIL, 2017).

Assim, o objetivo do presente relato foi descrever um caso de parto distócico, seguido de intervenção cirúrgica para remoção dos filhotes em uma cabra mestiça Anglo- Nubiana.

\section{RELATO DE CASO}

Uma cabra mestiça Anglo-Nubiana multípara, de quatro anos de idade, escore corporal médio, e peso de $40 \mathrm{~kg}$ foi atendida no
Hospital Veterinário Antônio Francisco Fonzar da Faculdade de Ciências Agrárias de Andradina apresentando dificuldade ao parto.

$\mathrm{Na}$ anamnese, o proprietário relatou que o animal tinha atingido o terço final da gestação, entrando em trabalho de parto há aproximadamente nove horas antes da apresentação ao hospital, entretanto, sem sucesso. Informou ainda, que a cabra não havia demonstrado problema ao parto na gestação anterior, contudo sua irmã sim. A mesma era criada em sistema extensivo e possuía status de vacinação e de desverminação atualizados.

Ao exame físico, o animal estava em estação, consciente, inquieto, com apetite presente e bom estado corporal. Por exame vaginal foi verificado prolapso retal e vaginal, dilatação cervical insuficiente, além de contrações abdominais fracas, porém sem observação de feto e das membranas fetais, bem como ausência de elementos contaminantes ou odor fétido.

$\mathrm{O}$ animal foi submetido ao exame clínico completo, sendo verificadas temperatura retal $\left(39,5^{\circ} \mathrm{C}\right)$ frequência cardíaca $(100$ batimentos/min) e frequência respiratória (60 respirações/min). As membranas de mucosas aparentes ocular, nasal, bucal e vulvar apresentavam-se (úmidas, brilhante e rósea clara), o tempo de perfusão capilar foi inferior a dois segundos, estando todos os parâmetros dentro dos limites fisiológicos para espécie. Realizou-se também uma ultrassonografia uterina para avaliação das condições do feto e viabilidade fetal, constatando gestação gemelar. Pode-se confirmar apresentação fetal anormal.

Baseando-se em todas essas informações colhidas concluiu-se o diagnóstico de distocia devido ao mal posicionamento de um dos fetos, o mesmo apresentava-se posicionado de modo transversal a cérvix, tornando impossível sua expulsão, e consequentemente, impedindo a passagem do segundo, ambos maturos, porém sem reação ao parto, devido à baixa contração uterina. Diante disso, o plano terapêutico consistiu em remover os fetos por meio da realização de uma cesariana.

Após liberação e autorização por escrito do proprietário, ocorreu à preparação do animal para entrada no centro cirúrgico, para execução do procedimento. $O$ animal foi contido e em seguida realizou-se a tricotomia da região ventro lateral esquerda, assepsia da região operatória com uso da solução de iodo povidona 
(PVPI) a 10\% e colocação dos campos cirúrgicos. Como medicação pré-anestésica foi administrado o cloridrato de xilazina na dose de $0,05 \mathrm{mg} / \mathrm{kg}$ por via intravenosa e como procedimento anestésico utilizou-se anestesia local infiltrativa, sendo aplicado $40 \mathrm{~mL}$ de cloridrato de lidocaína $2 \%$ sem vasoconstritor, utilizando a técnica de "L invertido" na região do flanco esquerdo. Na anestesia epidural também foi utilizado $5 \mathrm{~mL}$ de cloridrato de lidocaína $2 \%$ sem vasoconstrictor no espaço epidural caudal.

Após a anestesia foi realizada assepsia do local, em seguida realizada a diérese com uso do bisturi, atingindo primeiramente a pele, com uma incisão de aproximadamente $15 \mathrm{~cm}$ em sentido vertical, em seguida as camadas musculares externa e interna e posteriormente o peritônio, ocorrendo a exposição dos cornos uterinos. Em cada um deles foram realizadas incisões em sua curvatura maior, em uma área sem cotilédones, permitindo a retirada dos fetos ainda com vida. Os mesmos apresentavam-se maturos, sem sinal de sofrimento aparente.

Entretanto, após alguns instantes os mesmos vieram a óbito, mesmo sendo adotado manobras cardiovasculares, como massagem em região torácica para reanimação. Não foi realizada necropsia dos fetos, levando em consideração como o principal fator da causa mortis o tempo em que os fetos permaneceram mal posicionados, sem auxílio profissional ao parto.

Procedeu-se a lavagem do útero com solução fisiológica em temperatura ambiente para retirar todos os coágulos sanguíneos para evitar a formação de adesão. Em seguida foi realizada a síntese da parede uterina com fio catgut número 3.0 , praticando a sutura padrão simples separado. O útero foi reposicionado na cavidade abdominal e o líquido amniótico presente sobre a mesma foi removido com auxílio de compressas. Para a síntese da musculatura e pele utilizou-se fio de nylon agulhado número $0 \mathrm{em}$ padrão simples interrompido.

Realizou-se terapia antiinflamatória a base de Flunixin Meglumine na dose de $2 \mathrm{mg} / \mathrm{kg}$ por dia, via intramuscular, durante três dias. $\mathrm{Na}$ antibioticoterapia utilizou-se Oxitetraciclina $20 \%$ na dose de $20 \mathrm{mg} / \mathrm{kg}$ de peso vivo, em uma única aplicação e Penicilina Benzatina 20.000 $\mathrm{UI} / \mathrm{kg}$ a cada 48 horas, sendo realizada cinco aplicações, ambas administrada por via intramuscular profunda. O curativo local foi realizado com pomada a base de gentamicina, sulfadiazina de prata, vitamina $A$ e unguento.

Após a cirurgia o animal permaneceu por 10 dias no hospital veterinário sob observação, não apresentando nenhuma complicação pósoperatória neste período. Após 15 dias do procedimento foram removidas as suturas da pele e concedida a alta. A mesma foi reavaliada e compravado que não houve comprometimento em seu aparelho reprodutivo que possa interferir em sua vida reprodutiva futura.

\section{DISCUSSÃO}

A distocia em pequenos ruminantes ocorre geralmente devido a efeitos maternos ou fetais, sendo a dilatação cervical incompleta da mãe e o mal posicionamento do feto as principais causas desse problema em rebanhos de ovelhas e cabras (PUGH; BAIRD, 2012).

Em pequenos ruminantes, a posição normal do feto para saída pelo canal do parto é a cranial longitudinal, posicionado dorsosacralmente com as extremidades estendidas (BRAUN JR., 2007). No presente trabalho, a apresentação anormal de um dos fetos caracterizada por posição transversal dificultou sua passagem normal, e consequentemente impedindo a passagem do segundo, tornando a principal razão da distocia.

Como os fetos não estavam envolvidos no canal pélvico e para evitar maiores lesões no útero, bem como o tempo decorrido pela demora do produtor em buscar serviço veterinário, optou-se pela realização de uma cesariana como procedimento obstétrico na tentativa de preservar a vida da parturiente e de seus filhotes. Ressalta-se que em caprinos, a fragilidade do aparelho reprodutor é maior se comparado aos ovinos, dificultando assim algumas manobras obstétricas (SUNDARAVADANAN et al., 1996).

Segundo Hussain e Zaid (2010), a falha na expulsão do feto, progresso do parto dentro do tempo limite esperado para a espécie, chegando à completa inabilidade de parir, associada ao tempo de apresentação do animal ao atendimento veterinário e consequentemente ao tratamento da distocia podem causar a morte do filhote. Pois umas das principais consequências em quadros prolongadas é o desenvolvimento de metrite necrótica, geralmente fatal para o filhote (CHRISTOS et al., 2012). 
Assim, salienta-se que a maioria dos recém nascidos oriundos de cabras submetidas a cesárea geralmente não sobrevivem. Em estudo realizado por Ismail (2017), para a avaliar os principais fatores envolvidos nos quadros de distocia e cesárea em pequenos ruminantes observou que a chance de sobrevida neonatal ainda é muito baixa (23\%), devido principalmente as limitações fisiológicas dos mesmos. Daí a importância do treinamento dos trabalhadores das propriedades em geral para aumentar a taxa de sobrevivência dos filhotes.

No presente relato pode-se observar que o tempo em que o proprietário levou para procurar auxílio médico veterinário, foi possivelmente um dos fatores mais relevantes para a morte dos filhotes. Conforme Câmara et al. (2012), a taxa de mortalidade das crias será maior quanto mais demorado for o tempo decorrido entre o início do trabalho de parto e a procura por atendimento clínico adequado, em virtude na maioria das vezes por conduta inapropriada do proprietário.

De acordo com Kumar et al. (2013), o primeiro apontamento na maioria dos casos é salvar a vida da mãe, devido sua produtividade de carne, leite e descendentes, depois a vida dos filhotes, sendo assim, muitos pacientes são encaminhados para cesárea com este intuito. Portanto, em casos de parto distócico, há necessidade de procurar com urgência assistência obstétrica veterinária, visto que a distocia é responsável por grandes perdas econômicas em virtude da morte perinatal dos filhotes, observada na maioria destes casos (SHARMA et al., 2014).

Dessa forma, acredita-se que a conduta e os procedimentos realizados apresentaram resultados satisfatórios, sendo aceitável um prognóstico favorável para a fertilidade futura e performance reprodutiva da fêmea atendida. Corroborando Brounts et al. (1994), que afirmam que a cesárea é um método terapêutico eficaz em casos de distocia em ovinos e caprinos, além de não afetar negativamente a fertilidade subsequente das fêmeas.

\section{CONCLUSÃO}

$O$ procedimento e manipulação da cirurgia foram bem-sucedidos, visto que a cabra apresentou total recuperação não ocorrendo complicações secundárias, demonstrando assim o resultado satisfatório com a intervenção obstétrica.

\section{REFERÊNCIAS}

BHATTACHARYYA, H.K.; FAZILI, M.R.; BHAT, F.A.; BUCHOO, B.A. Prevalence and dystocia of sheep and goats: A study of 70 cases (2004-2011). Journal of Advanced Veterinary Research, v.5, n.1, p.14-20. 2015.

BLOOD, D.C.; STUDDERT, V.P.; GAY, C.C. Saunders Comprehensive Veterinary Dictionary. 4. ed. London: Saunders, 2011.

BRAUN JR., W. Parturition and Dystocia in the Goat. In: YOUNGQUIST, R.S.; THRELFALL, W.R. Current Therapy in Large Animal Theriogenology. 2. ed. Saint Louis: Saunders, 2007. p.556-558.

BROUNTS, S.H.; HAWKINS, J.F.; BAIRD, A.N.; GLICKMAN, L.T. Outcome and subsequent fertility of sheep and goats undergoing cesarean section because of dystocia: 110 cases (1981-2001). Journal of the American Veterinary Medical Association, v.224, n.2, p.275-279, 2004. https://doi.org/10.2460/javma.2004.224.275

CÂMARA, A.C.L.; DANTAS, A.C.; GUIMARÃES, J.A.; AFONSO, J.A.B.; SOUZA, M.I.; COSTA, N.A.; MENDONÇA, C.L. Análise dos fatores relacionados a 26 casos de distocia em cabras no agreste e sertão de Pernambuco. Veterinária e Zootecnia, v.19, n.2, 2012.

CHRISTOS, N.B.; LAZARIDIS, L.; KARAGIANNIS, I.; KIOSSIS, E.; TSOUSIS, G.; PSYCHAS, V.; NEKTARIOS, D.G. Prolonged dystocia, uterine necrosis, and ovariohysterectomy in a Chios ewe. Turkish Journal of Veterinary and Animal Sciences, v.36, p.211-221, 2012. doi:10.3906/vet1105-11

HUSSAIN, S.O.; ZAID, N.W. Dystocia in goats, causes and treatment. Al-Qadisiyah Journal of Veterinary Medicine Sciences, v.9, n.1, p.63-67, 2010.

ISMAIL, Z.B. Dystocia in sheep and goats: outcome and fertility following surgical and nonsurgical management. Macedonian Veterinary Review, v.40, n.1, p.91-96, 2017. http://dx.doi.org/10.1515/macvetrev-2017$\underline{0012}$ 
KUMAR, V.; TALEKAR, S.H.; AHMAD, R.A.; MATHEW, D.D.; ZAMA, M.M.S. Delayed cases of dystocia in small ruminants - etiology and surgical management. The Indian Journal of Veterinary Science, v.1, n.1, p.47-54, 2013.

MAJEED, A.F.; TAHA, M.B.; AZAWI, O.I. Cesarean section in Iraqi Awassi ewes: A case study. Theriogenology, v.40, n.2, p.435-439, 1993. https://doi.org/10.1016/0093-691X(93)90280-I

PUGH, D. G.; BAIRD, N.N. Sheep and goat medicine. 2. ed. Saint Louis: Elsevier, 2012.

SHARMA, A.; KUMAR, P.; SINGH, M.; VASISHTA, N. Retrospective analysis of dystocia in small ruminants. Intas Polivet, v.15, n.2, p.287-289, 2014.

SUNDARAVADANAN, V.K.; SHIVAPRAKLASH, B.V.; HONNAPPAGOL, S.S. Ring womb and uterine rupture in a goat. Indian Veterinary Journal, v.73, n.3, p.324-325, 1996.

Recebido para publicação em 03/08/2017

Revisado em 18/05/2018

Aceito em 03/07/2018 